\title{
Micromorphological analysis and bond strength comparison of two adhesives for different degrees of dental fluorosis
}

\author{
Shuangfeng Liu' ${ }^{1,2}$ Yanxia Zhu² and Tana Gegen ${ }^{2 *}$
}

\author{
*Correspondence: \\ endotana@163.com \\ ${ }^{2}$ The Department \\ of Stomatology, Affiliated \\ Hospital of Inner Mongolia \\ Medical University, \\ Hohhot 010000, China \\ Full list of author information \\ is available at the end of the \\ article
}

\begin{abstract}
The objective of this study was to analyze morphologically the all-etching bonding system and self-etching bonding system for enamel with different degrees of fluorosis and evaluate the bond strength of each system. Teeth that were indicated for extraction owing to orthodontic or periodontal problems were selected. According to Dean's index and the Thylstrup-Fejerskov index, 180 extracted teeth were divided into three groups of mild, moderate, and severe dental fluorosis (DF), with 60 teeth in each group. The teeth in each group were randomly divided into two subgroups $(n=30)$, which were then subjected to the all-etching bonding system (Prime \& Bond NT) and selfetching bonding system (SE-Bond). Each group of adhesives was used to bond Z350 universal resin (3 M) to the etched dental enamel. Tensile and shear tests were conducted to determine the bond strength. Subsequently, the fractured specimens were investigated using scanning electron microscopy (SEM) and confocal laser scanning microscopy (CLSM). The Prime \& Bond NT was statistically significant for the tensile and shear strength of enamel with mild fluorosis $(P<0.05)$ but did not exhibit a significant difference for moderate and severe DF $(P>0.05)$. The SE-Bond was not statistically significant for the tensile and shear strength of mild, moderate, or severe DF $(P>0.05)$. The SEM and CLSM results reveal that the mild fluorosis enamel crystals were relatively dense, and a small amount of resin remained. The moderate fluorosis enamel crystals were loosely arranged, and the gaps were widened. The severe fluorosis enamel crystals were irregularly arranged. The disorder was aggravated, and the dentinal orifice was exposed by partial enamel exfoliation. The bonding strength of mild fluorosis enamel with the Prime \& Bond NT was better than that with the SE-Bond, and cohesive failure was the most common mode of failure. Because there was no difference in the bonding strength of the SE-Bond for different degrees of DF, we recommend the use of the all-etching adhesive system in the clinical treatment of teeth with mild fluorosis.
\end{abstract}

Keywords: Dental fluorosis, Enamel, Tensile bond strength, Shear bond strength, CLSM

\section{Introduction}

In 1936, it was shown that an increase in fluoride content in water causes dental fluorosis (DF), which is an alteration of the tooth enamel and can be observed as spots ranging from a whitish to dark brown color. In severe cases, this leads to the loss of tooth enamel

(c) The Author(s) 2020. This article is licensed under a Creative Commons Attribution 4.0 International License, which permits use, sharing adaptation, distribution and reproduction in any medium or format, as long as you give appropriate credit to the original author(s) and the source, provide a link to the Creative Commons licence, and indicate if changes were made. The images or other third party material in this article are included in the article's Creative Commons licence, unless indicated otherwise in a credit line to the material. If material is not included in the article's Creative Commons licence and your intended use is not permitted by statutory regulation or exceeds the permitted use, you will need to obtain permission directly from the copyright holder. To view a copy of this licence, visit http://creativeco mmons.org/licenses/by/4.0/. 
[1]. Subsequently, the pathogenesis of DF was widely investigated, and it was found that exposure to high-fluorine potable water and the absorption of fluoride through food causes DF [2] owing to the excessive consumption of fluorine during the development of teeth with abnormal surface morphology. Moreover, DF is a common and prominent disease in the early stages of chronic fluorosis and can also lead to skeletal fluorosis in severe cases, which, in turn, results in brittle bones that are vulnerable to tensile forces [3].

The fluorosis of the enamel is characterized by reduced mineral content, which results in surface and subsurface porosities and subsequent visual and physical changes [4]. Although most developed countries have effectively solved these public health issues and the quality of water has improved, various developing countries still have to treat DF patients. Clinically, DF compromises the tooth aesthetics, and visible symptoms range from narrow white lines following the perikymata to discrete white opaque areas or even an entirely chalky-white tooth surface, depending on the severity [5]. In some patients, the enamel may become so porous that the outer layers break down and the exposed porous subsurface becomes discolored from a light to dark brown color [6]. The appearance of this discoloration and the pitting of the enamel surface may be aesthetically objectionable to the extent that the patient may experience social repercussions.

Various treatments and management strategies, such as bleaching, microabrasion, composite restorations, veneering, crowning, or a combination of two approaches, have been proposed to alleviate the effects of DF [5, 7-9]. However, owing to the physical and morphological changes induced by DF, bonding to this substrate is still clinically challenging because fluorapatite is more resistant to acid dissolution compared with hydroxyapatite [10]. Although good results have been obtained for sound enamel, there is no consensus with regard to the application of phosphoric acid to fluorotic enamel [11]. Various studies have demonstrated that, owing to the particular enamel structure of DF, the micromechanical fitting after acid etching is not satisfactory, which affects the bonding between the adhesive and the enamel $[12,13]$.

The objective of this study was to use tensile and shear testing to evaluate the bonding strength of the all-etching bond system and self-etching bond system in vitro for different degrees of DF. Additionally, stereoscopic microscopy and confocal laser scanning microscopy (CLSM) were used to observe the ultrastructure of the bonding interface. The results obtained by this study can provide clinicians with relevant data to inform their selection of highly effective adhesive systems for treating different degrees of DF.

\section{Materials and methods}

\section{Sample collection and processing}

The study protocol was approved by the Ethics Committee of the Affiliated Hospital, Inner Mongolia Medical. The samples were collected from subjects who provided written informed consent to participate in the study. The 30 samples from the control group were obtained from healthy subjects undergoing permanent tooth extraction for orthodontic purposes. The DF teeth were free of caries and had been extracted for periodontal reasons. The exclusion criteria are as follows: (1) The surface had cracks and caries; (2) DF had received any treatment; (3) DF had been soaked in hydrogen peroxide. Informed consent to use the extracted teeth for research purposes was 
obtained from the subjects before the extraction. The DF degree was diagnosed by applying Dean's index (DI) [14] and the Thylstrup-Fejerskov Index (TFI) [4, 6, 15] [TFI: 0 , healthy enamel; $1-3$, mild fluorosis; $4-5$, moderate fluorosis; $6-9$, severe fluorosis]. The 180 extracted DF teeth were divided into three groups of mild, moderate, and severe DF, with 60 teeth in each group. The teeth in each group were randomly divided into two subgroups $(\mathrm{n}=30)$, which were subjected to the all-etching bonding system (Prime \& Bond NT) and self-etching bonding system (SE-Bond). Before preparation, the teeth were washed with running water, and soft tissue was removed from the tooth surface using a surgical blade. All treated teeth were immersed in $1 \%$ Chloramine and stored in a refrigerator at $4{ }^{\circ} \mathrm{C}$ for later use (Fig. 1).

\section{Preparation of experimental specimens}

The teeth were cleaned to remove any debris before being used in vitro. Superanhydrite was mixed to a certain proportion, filled in the prepared mold, and embedded in the DF root in vitro. Moreover, the crown above the boundary of the cementum was exposed. According to the designed grouping, the enamel surface of teeth with mild, moderate, and severe fluorosis was used to remove $0.5 \mathrm{~mm}$, and the polished interface was observed using stereoscopic microscopy $(32 \times)$ to ensure that the remaining tissue surface was still located in the enamel layer. Rectangular pores with a size of $2 \times 3 \mathrm{~mm}$ were made on one side of the tape using a hole puncher. The porous tape was pasted onto the enamel grinding area, and was then used to bond with the alletching bonding system (Prime \& Bond NT) and self-etching bonding system (SEBond), respectively, according to the manufacturer's recommendations. The details of the materials used in this study are summarized in Table 1. Each group of adhesives was used to bond Z350 universal resin $(3 \mathrm{M})$ to the etched dental enamel. The composite resin (Z350) was filled into the layers under pressure and cured, and a resin

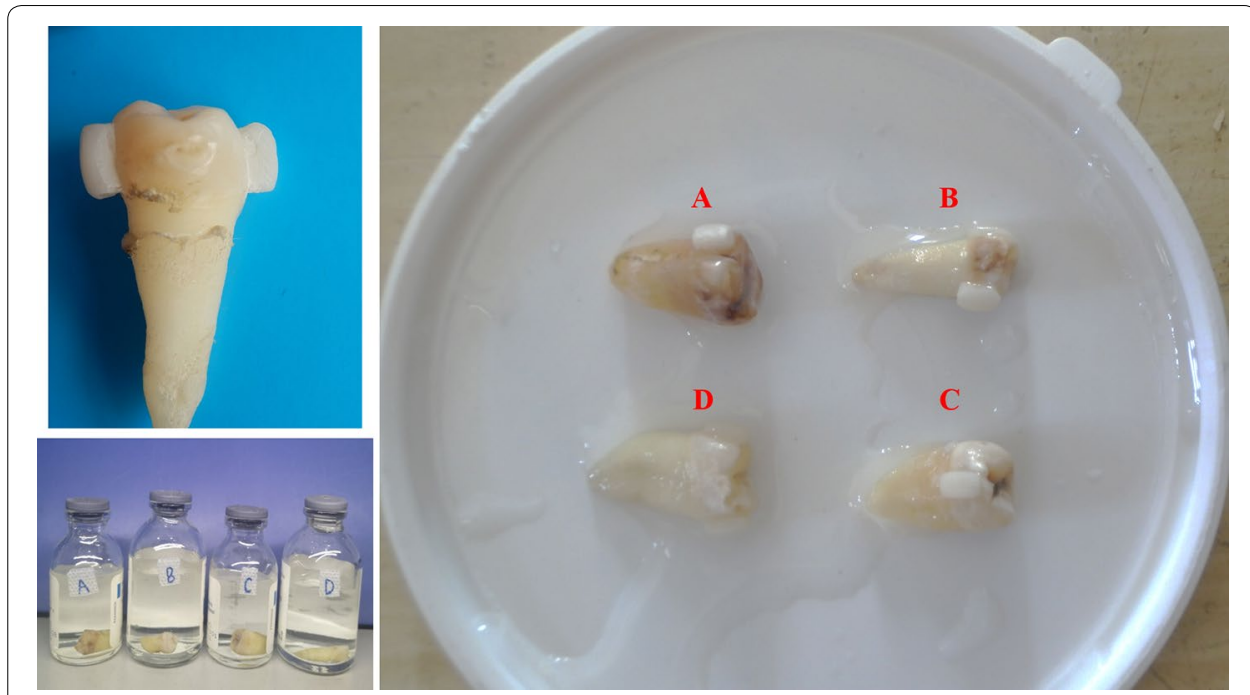

Fig. 1 Preparation of experimental specimens: (a) Severe DF; (b) Moderate DF; (c) Mild DF; (d) Normal healthy teeth 
Table 1 Materials, manufacturers, and the components, of the enamal bonding agents used in this study

\begin{tabular}{|c|c|c|c|}
\hline Adhesive system & Manufacturer batch & Composition & Directions \\
\hline Prime \& Bond NT & Densply,DeTrey Gmb H, USA & $\begin{array}{l}\text { Etch-35\% phosphoric acid Di-and } \\
\text { Trimethacrylate resins } \\
\text { PENTA, Amorphous Silicon } \\
\text { Dioxide } \\
\text { Cetylamine hydrofluoride, } \\
\text { Acetone } \\
\text { Phosphoric Acid } \\
\text { Highly dispensed silicon dioxide } \\
\text { Colorant } \\
\text { Water }\end{array}$ & $\begin{array}{l}\text { Etch for } 15 \mathrm{~s} \text {; Etch } \\
\text { wash and blot dry; } \\
\text { Apply two coats of } \\
\text { adhesive; } \\
\text { Gently drying } 5 \mathrm{~s} \text {; } \\
\text { Light cure for } 10 \mathrm{~s}\end{array}$ \\
\hline Clearfil SE Bond & $\begin{array}{l}\text { Kuraray Medical, } \\
\text { Tokyo, Japan }\end{array}$ & $\begin{array}{l}\text { Primer-MDP, EMA, hydrophilic } \\
\text { dimethacrylate,photoinitiator, } \\
\text { water } \\
\text { Bond-MDP, HEMA, hydrophilic } \\
\text { dimethacrylate, Bis-GMA, } \\
\text { microfiller }\end{array}$ & $\begin{array}{l}\text { Apply primer for } 20 \mathrm{~s} \text {; } \\
\text { Blow air gently; } \\
\text { Apply bonding } \\
\text { agent; } \\
\text { Light cure for } 10 \mathrm{~s}\end{array}$ \\
\hline
\end{tabular}

MDP 10-mehtacryloyloxydecyl dihydrogen phosphate, PENTA dipentaerythritol penta acrylate monophosphate, Bis-GMA bisphenol A-glycidyl methacrylate

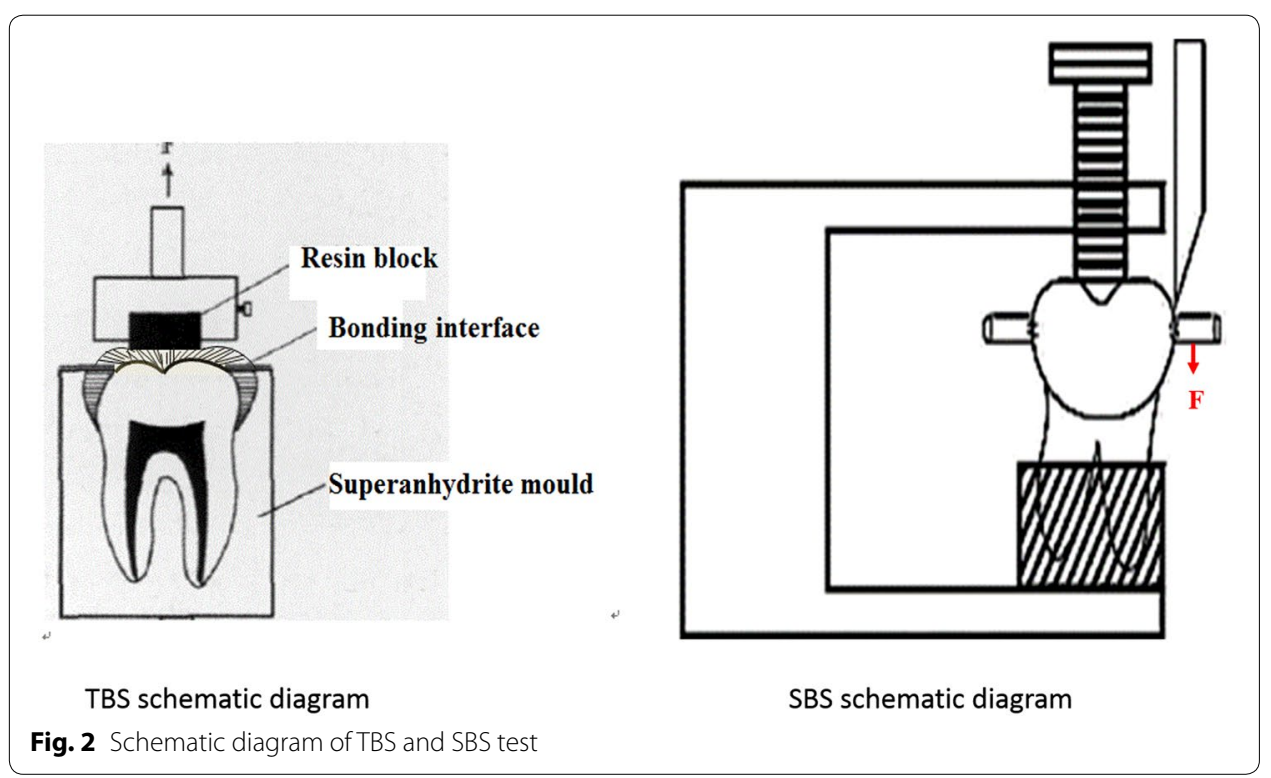

block with an area of $2 \times 3 \times 2 \mathrm{~mm}^{3}$ finally formed. The prepared experimental specimens were kept in water and stored in a refrigerator at $4{ }^{\circ} \mathrm{C}$ for later use.

\section{Mechanical property test}

The prepared experimental specimens were fixed onto the Universal Capability Tester (WDW-100, China) with a fixture whose load head was aligned with the bonding surface. Additionally, a loading force was continuously applied with a horizontal tensile loading speed of $1.0 \mathrm{~mm} / \mathrm{min}$ until the test specimen bonding surface broke. The mean bond strengths were recorded from the maximum load at failure and converted to MPa. The actual bonding interface area was measured using a Vernier caliper (Fig. 2). 
The tensile strength and shear strength $(\mathrm{MPa})=$ Maximum breaking load $(\mathrm{N}) /$ Bonding area $\left(\mathrm{mm}^{2}\right)$.

\section{SEM observation of bonding surface}

After the specimen of the mechanical property test had completely dried, the teeth were cut sagittally from the buccal side to the lingual side, along the long axis of the tooth through the center of the specimen. The fracture surface of the test specimens was observed using scanning electron microscopy (SEM; Hitachi S-4800, magnification $5-100,000 \times)$ to investigate the fracture mode of the debonded specimens after the TBS test. The fracture mode was classified into four types: Type 1: adhesive/mixed (A/M); Type 2: cohesive-enamel (CE); Type 3: cohesive-resin (CR); Type 4: premature failure (PF).

\section{Confocal laser scanning microscopy (CLSM) observation}

After the specimen of the mechanical property test had completely dried, it was immersed in $1 \mathrm{~g} / \mathrm{L}$ rhodamine B fluorescent dye solution at $37^{\circ} \mathrm{C}$ for $24 \mathrm{~h}$. After rinsing with distilled water for $10 \mathrm{~s}$, the teeth were cut sagittally from the buccal side to the lingual side, along the long axis of the tooth and through the center of the specimen. The fracture surface of the test specimen was observed using CLSM (LEXT OLS4100, $500 \times$ magnification) at $100 \%$ relative humidity. The CLSM used in this study is a video rate instrument that can achieve a frame time of $33 \mathrm{~ms}$ by using an accousto-optic deflector for high-speed scanning on one axis. This allows the rapid three-dimensional assessment of samples, while avoiding damage caused by prolonged drying. The utility of this technique for imaging the depth and shape of lesions has been extensively demonstrated [16]. In this study, the depth of the different degrees of fluorosed enamel was evaluated from the surface of the undemineralized acid-resistant varnish-covered tooth part to the deepest demineralized front.

\section{Statistical analysis}

The data are expressed as the mean \pm standard deviation. Statistical analysis was carried out using SPSS software (version 17.0, IBM Corp., Armonk, NY, USA). The bond strengths were compared with the enamel type as a fixed factor and the bonding approach as a random factor using two-way ANOVA analysis of variance under the general linear model. The SNK- $q$ test was carried out to identify differences amongst the groups. Pearson's chi-squared test was used to investigate the association between the failure mode and the fluorosis type of each bonding approach. Before the analysis, the failure modes were categorized into four groups: A/M, CE, CR, and PF; $P<0.05$ was considered to be statistically significant.

\section{Results}

\section{Tensile bonding strength (TBS)}

For the all-etching adhesive, there were significant differences in the tensile bonding strength of the mild, moderate, and severe DF $(P<0.05)$. However, for the selfetching adhesive, there were no significant differences in the tensile strength of the mild, moderate, and severe DF $(P>0.05)$. By comparing the tensile strength for the 


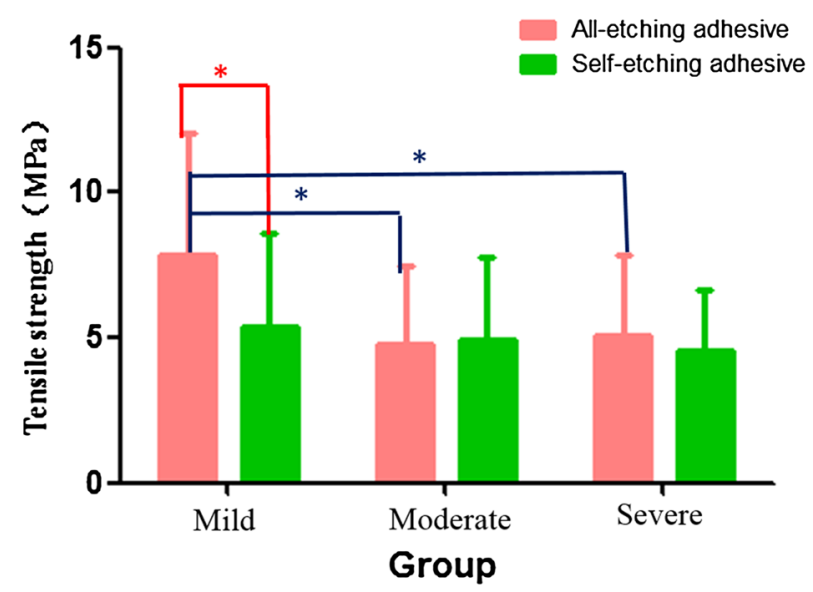

Fig. 3 Histogram analysis of tensile strength determination

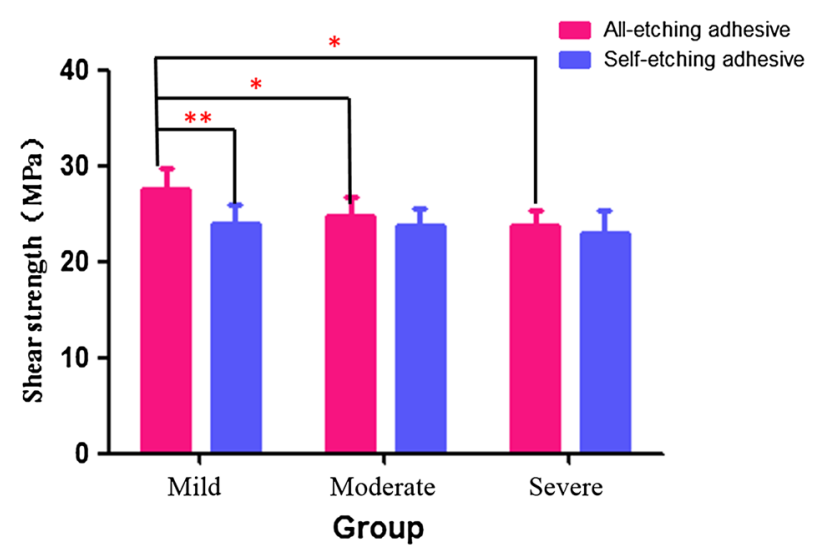

Fig. 4 Histogram analysis of shear strength determination

same degree of DF and with different adhesives, it was found that, with the all-etching adhesive, the tensile strength for mild DF was significantly higher than that with the self-etching adhesive $(P<0.05)$. Additionally, with the all-etching adhesive and self-etching adhesive, there was no significant difference between the moderate and severe DF $(P>0.05)$ (Fig. 3).

\section{Shear bonding strength(SBS)}

The all-etching adhesive had significant differences in the shear bonding strength of the mild, moderate, and severe DF $(P<0.05)$. For the self-etching adhesive, there was no significant difference in the shear strength of the mild, moderate, and severe DF $(P>0.05)$. By comparing the shear strength of different adhesives for the same degree of DF, it was found that, with the all-etching adhesive, the shear bonding strength of the mild DF was significantly higher than that achieved by the self-etching adhesive $(P<0.05)$. Additionally, with the all-etching adhesive and self-etching adhesive, there was no significant difference between the moderate and severe DF $(P>0.05)$ (Fig. 4). 


\section{Observation of fracture interface using SEM}

The SEM results obtained in this study reveal that the enamel of mild DF was relatively uniform and dense, and a small number of crystals were disorderly arranged. The interspace of the crystals was slightly widened, and various amounts of adhesive residue were visible. This indicates that, under the influence of force, fracture occurred between the resin and the enamel surface. In other words, interface fracture occurred. The bonding strength of mild DF is determined by the adhesive. The enamel on the surface of severe fluorosis was severely exfoliated, and even some of the dentin tubules were exposed. The enamel was relatively loose and non-uniform (Figs. 5 and 6 ). The fracture forms of the two adhesives were mainly cohesive-enamel, and a statistical difference did not exist between the two groups $\left(\chi^{2}=1.660, P>0.05\right)$ (Table 2$)$.

This indicates that the enamel strength of severe DF is low. Additionally, enamel exfoliation occurred under the influence of force, which led to enamel fracture. Thus, the bonding strength of severe DF is determined by the enamel strength. The moderate DF was similar to severe fluorosis, and the enamel was also exfoliated under force. However, the degree of enamel exfoliation was lower than that of severe fluorosis. The electron microscopy results are essentially consistent with the results of the mechanical property test.
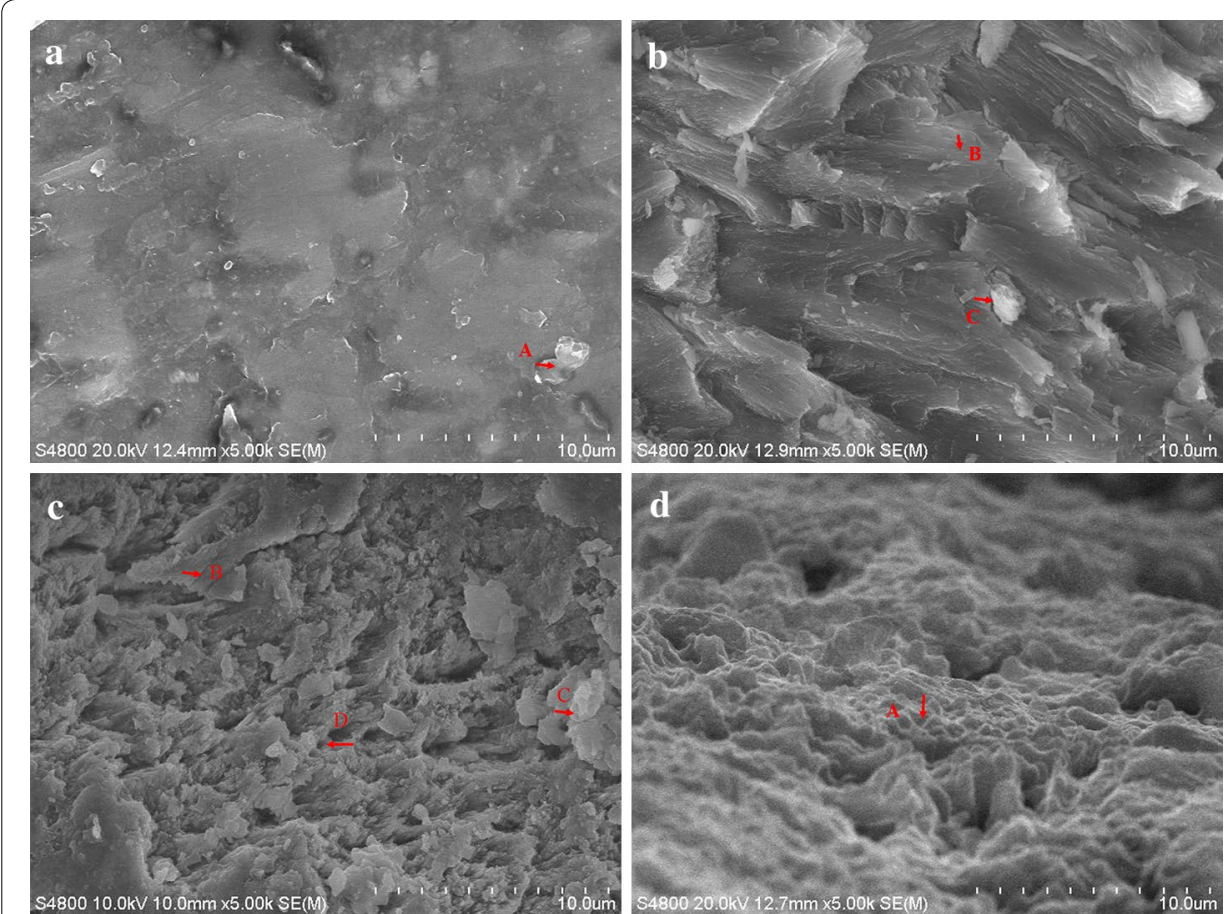

Fig. 5 SEM results for mild, moderate, and severe DF interface fracture after all-etching adhesive strength test (original magnification, $5.00 \mathrm{k} \times$; scale bar $=10.0 \mu \mathrm{m}$ ): a Fracture interface of mild fluorosis with more adhesive residue in bond interface.(b Fracture interface of moderate fluorosis; a small amount of adhesive remained, while the dentin tubules were partly exposed. c Fracture interface of severe fluorosis; the enamel was completely exfoliated, the dentin tubules were completely exposed, and the crystals were disordered; the interspace between the crystals was significantly widened, and the number of enamel columns was small. d Normal healthy enamel bonding group as control group 

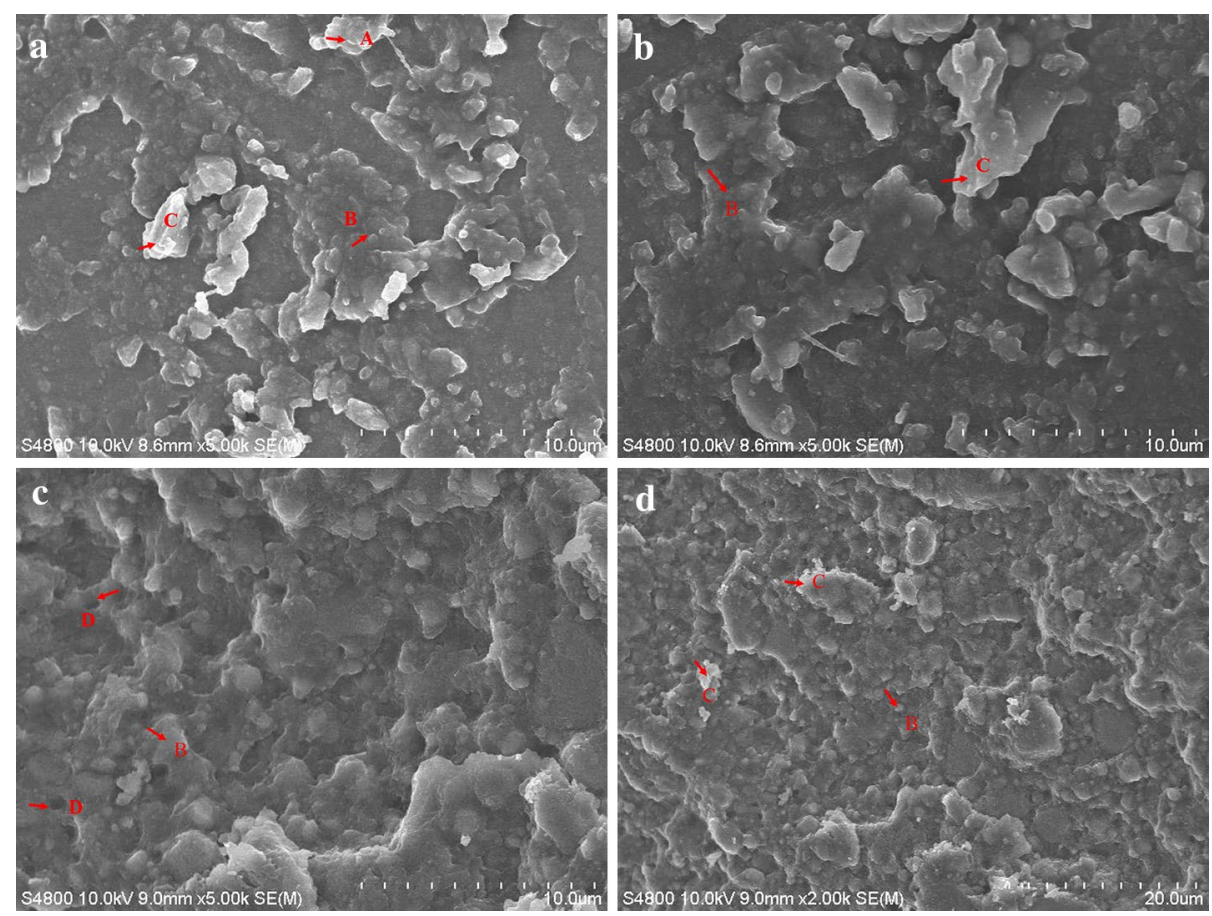

Fig. 6 SEM results for mild, moderate, and severe DF interface fracture after self-etching adhesive strength test (original magnification, $5.00 \mathrm{k} \times$; scale bar $=10.0 \mu \mathrm{m}$ ): a Fracture interface of mild fluorosis; the enamel of the bonding surface was relatively uniform and dense, a small number of crystals were disorderly arranged, the interspace between the crystals was slightly widened, and a small amount of adhesive remained. $\mathbf{b}$ Fracture interface of moderate fluorosis; the enamel was exfoliated under tension lower than that of severe fluorosis. c Fracture interface of severe fluorosis; the surface enamel was severely exfoliated, and even some dentin tubules were exposed. $\mathbf{d}$ Normal healthy enamel bonding group as control group

Table 2 Number of specimens (\%) according to fracture mode for Fluorotic enamel

\begin{tabular}{lllll}
\hline Group & A/M & CE & CR & PF \\
\hline Prime \& Bond NT & 3 & 26 & 1 & 0 \\
Clearfil SE Bond & 4 & 23 & 2 & 1 \\
\hline
\end{tabular}

$A / M$ adhesive/mixed, $C E$ cohesive-enamel, $C R$ cohesive-resin, $P F$ premature failure

\section{Observation of fracture interface using CLSM}

The results obtained by this study reveal that, as the DF degree increased, the enamel crystal was more loosely arranged. Moreover, after the all-etching adhesive bonding process, the bonding interface was rougher than that of the self-etching adhesive. Additionally, the bonding interface appeared more uneven (Figs. 7 and 8).

\section{Discussion}

Fluoride is needed for the normal development of bone and teeth. At high levels, however, fluoride affects the development of teeth and bone. Specifically, DF is caused by the ingestion of excess fluoride mainly through the drinking of water $[17,18]$. Additionally, the use of fluoride is related to the exacerbation of DF. Water fluorination 


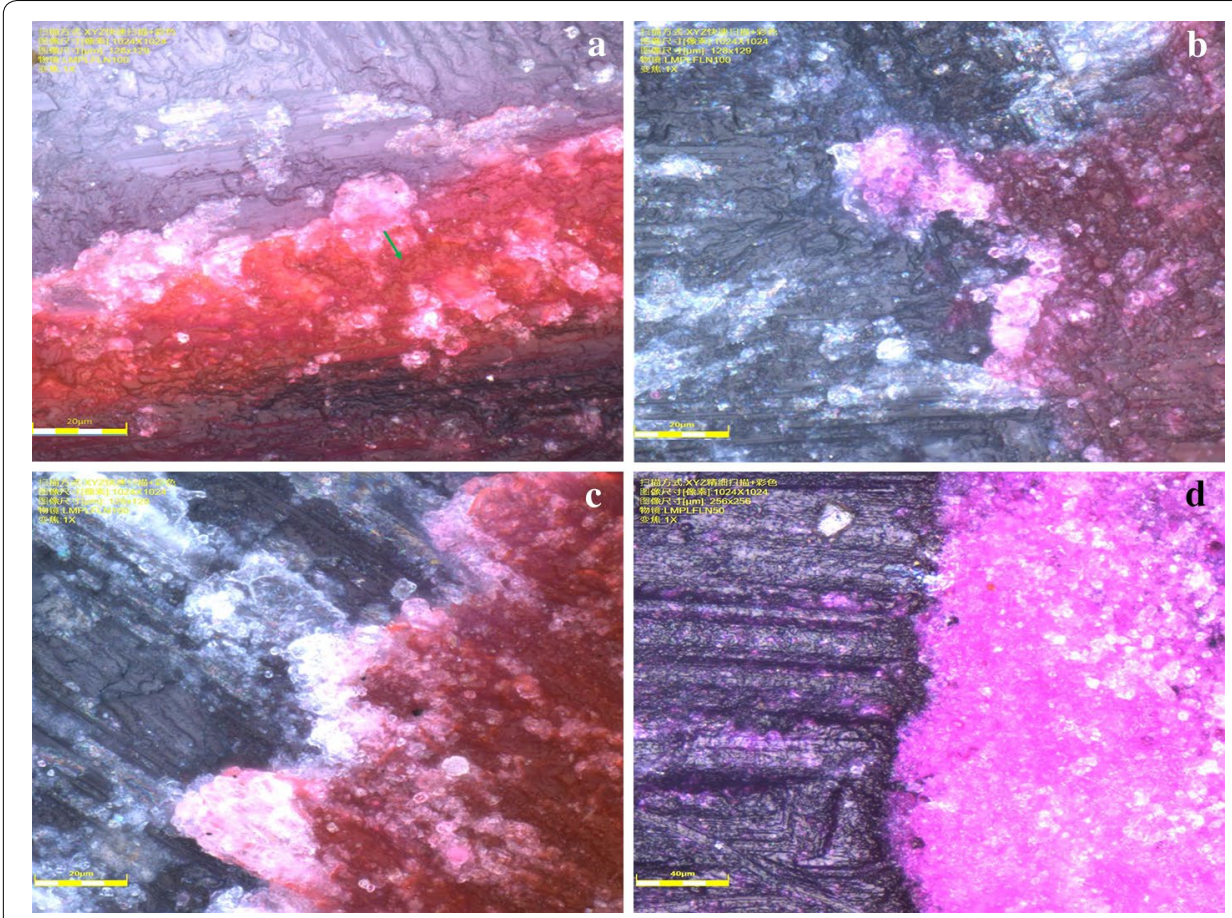

Fig. 7 CLSM results for mild, moderate, and severe DF fracture interface after all-etching adhesive strength test (original magnification, $100 \times$; scale bar $=20 \mu \mathrm{m}$ ): a Fracture interface of mild fluorosis; $\mathbf{b}$ Fracture interface of moderate fluorosis; $\mathbf{c}$ Fracture interface of severe fluorosis; $\mathbf{d}$ Normal healthy enamel bonding group as control group

and the use of fluoride products, such as fluoride toothpaste, specific food/beverages, and fluoride supplements, are considered to be risk factors for the occurrence of DF [1].

In fluorosed teeth, the highly mineralized enamel surface layer comprises a mixture of many large and extremely small crystals, whereas the hypomineralized subsurface area comprises fairly sparsely arranged large crystals with a few small crystals. Some of the crystals in the subsurface hypomineralized layer exhibit defects such as perforations [19]. In more severe cases, the hypomineralization is extensive, and the outer well-mineralized surface layer is rather brittle. Thus, chewing forces may result in the formation of surface enamel defects, which appear as single pits along the perikymata. Alternatively, the surface enamel may be chipped away, corresponding to incisal edges or cuspal tips. Particularly, the occlusal surfaces are rapidly worn, often to such an extent that the hypomineralized porous layer is abraded away [20].

Presently, with regard to dental enamel bonding theory, various scholars hold that a mechanical chimera, physical adsorption, and chemical bonding are the main means of dental enamel bonding [21]. Because the retention force between the adhesive and the enamel mainly depends on mechanical fitting, the surface morphology of the enamel has a certain effect on the bonding strength. Most studies on the adhesive strength of teeth have considered healthy teeth. However, the DF enamel is different from the crystal enamel structure of normal teeth [17]. This study demonstrated that the adhesive and dental enamel are firmly held, mainly by the mechanical fitting of the micropores 


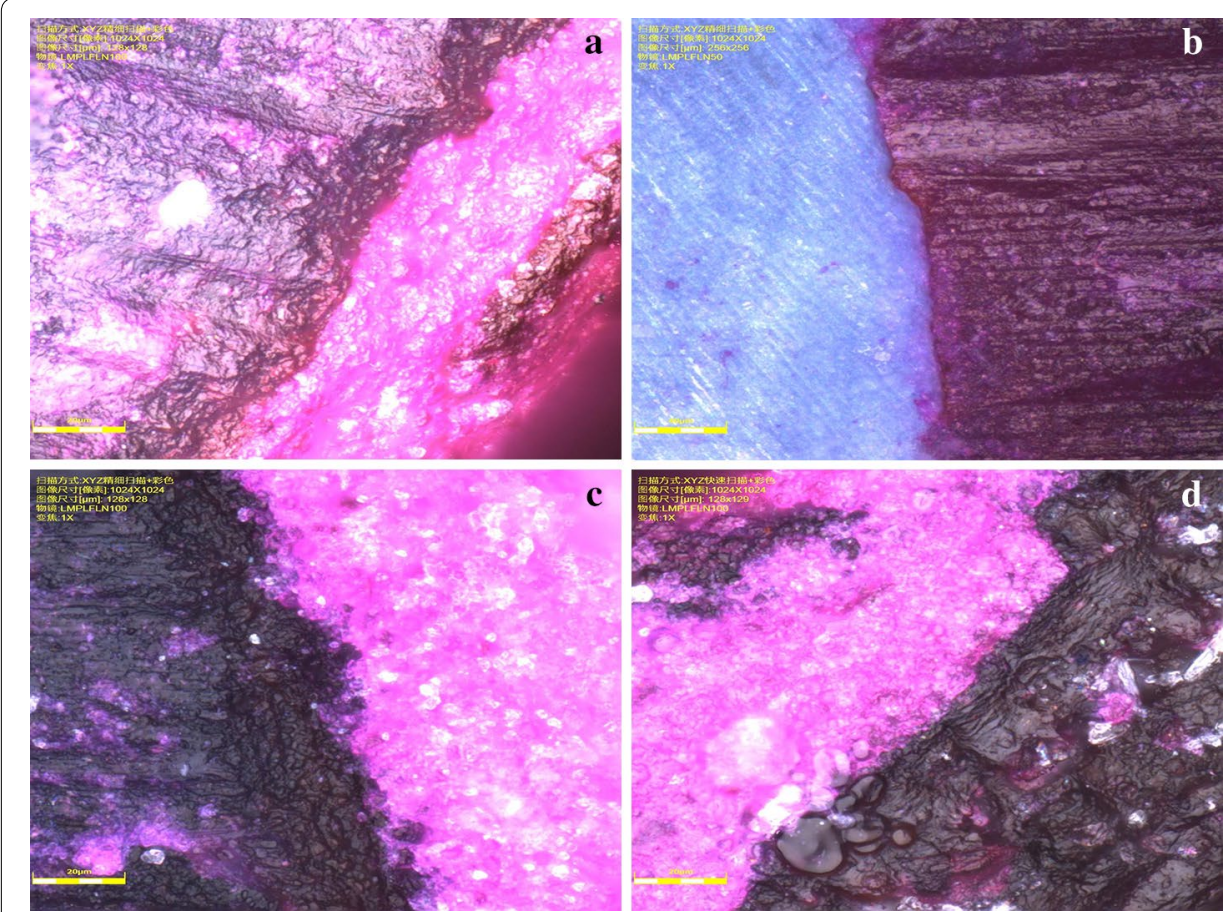

Fig. 8 CLSM results for mild, moderate, and severe DF interface fracture after self-etching adhesive strength test (original magnification, $100 \times$; scale bar $=20 \mu \mathrm{m}$ ): a Fracture interface of mild fluorosis; $\mathbf{b}$ Fracture interface of moderate fluorosis; $\mathbf{c}$ Fracture interface of severe fluorosis; $\mathbf{d}$ Normal healthy enamel bonding group as control group

produced by enamel acid etching, as observed using CLSM. Hence, the relationship between the physical state of the enamel surface and the bond strength after acid etching is very important.

By observing the fracture interface of a mildly fluorosed enamel using SEM, it was found that the distinct dissolution of the outer surface exhibited many obvious surface irregularities such as shallow fissures and other defects. For moderately and severely fluorinated enamels, the loss of the external microscopic surface was visible and some dentin tubules had been exposed. These results are consistent with the results obtained by various previous studies. Although fluorapatite may be more acid-resistant, other structural changes, such as the fluorotic enamel's outer hypermineralized layer with a porous hypomineralized subsurface and low surface energy, impair the surface wetting, which results in the decrease in bond strength [22].

In this study, the interface fracture was observed using SEM, and cohesive failure was the most common mode of failure. Moreover, the fractured specimens were observed using CLSM, and cohesive failure was the most prevalent mode of failure in moderate and severely fluorosed teeth that had previously undergone phosphoric acid etching. This observation should be considered because the self-etching primer adhesive system produces a predominant adhesive failure [23]. Notably, various studies have demonstrated that most specimens (98\%) exhibit adhesive/mixed failures, with $1 \%$ exhibiting premature failure and $1 \%$ exhibiting cohesive failure exclusively within the enamel or cohesive failure exclusively within the resin composite in DF [6]. 
By comparing the sound and fluorotic enamel, the lower bond strengths were obtained for the fluorotic enamel, regardless of the application technique. These results should be investigated further because the fluorotic enamel is more resistant to acidic dissolution owing to the presence of fluorapatite, which adversely affects the adhesive performance [10]. Various studies [24] have reported that the enamel of DF produces micropores owing to the effect of acid etching and decalcification. As the fluorosis severity increases, the subsurface enamel along the tooth becomes increasingly porous (hypomineralized), and the lesion extends toward the inner enamel [25].

From the viewpoint of mechanical bonding [24], it is inferred that, if the glazed column of the glazed surface is perpendicular to the bonding surface after acid treatment, it can produce an ideal honeycomb structure with maximum adhesion. Additionally, if the partial glaze column is parallel to the bonding surface after acid treatment, it can produce minimum adhesion. As has been confirmed, the abnormal enamel morphology, whether it is the collapse of the enamel development or the disorder of the glaze column, affects the bonding strength. Moreover, it has been reported that the enamel surface of fluorosed teeth appears highly uneven and rough, and exhibits cracks and fissures. The enamel surface exhibits pits with variable dimensions in the discolored area of the teeth, which appear as punched lesions on the enamel surface and thus expose the underlying porous enamel [17]. A recent study on the ground sections of fluorosed teeth reported crescent-shaped hypomineralized areas in the enamel and increased interglobular dentin spaces [26].

This study found that, in tensile and shear testing, the all-etching bonding system for teeth with mild fluorosis had higher bonding strength compared with the self-etching bonding system, which is consistent with the results obtained by many previous studies. Owing to the particular enamel structure of the DF, the micromechanical fitting after acid etching is not satisfactory, and this affects the bonding effect between the adhesive and the enamel $[12,13]$. Self-etching adhesive systems use weak acidic monomers to completely condition the enamel/dentin substrate. Various previous studies [23] have demonstrated that K-etchant gel (37\% phosphoric acid) produces typical etching patterns consistent with phosphoric acid, regardless of the fluorosis severity. The etching pattern observed by a self-etching primer is shallower than that of the K-etchant, which uses MDP as the etching agent. This is attributed to a shift in the crystal orientation or the enlargement of laminar pores, which are considered as more important diffusion pathways, compared with the interprismatic enamel, during the acid attack [27]. Many studies have also reported that the two-step etch-and-rinse adhesive system used in the present study, namely, Single-Bond, uses 35\% phosphoric acid with a $\mathrm{pH}$ of approximately 0.6. Notably, $35 \%$ phosphoric acid is strong acid. The two-step self-etch adhesive, SE Bond, is classified as a mild self-etch adhesive with a $\mathrm{pH}$ of 2.0. Because self-etch adhesives are less acidic than phosphoric acid, they do not demineralize the enamel to the same extent. Thus, they yield a less micro-retentive surface and, consequently, lower bonding strength [28].

Shallow fissures with other irregularities have been observed in mildly fluorosed enamel [29]. The all-acid etching bonding system uses phosphoric acid to etch the tooth enamel before demineralization, and removes the stained layer through acid etching. Compared with the self-etching bonding system, the all-acid etching bonding can form a wider 
penetration area on the DF surface, and the formed resin protrusions are more uniform, which improves the effect of micromechanical fitting [12, 30, 31]. Various studies have reported that self-etching bonding systems are inferior to all-etching bonding systems when bonding is performed for moderately and severely fluorosed enamel. However, this is inconsistent with our results [11,31]. A more detailed examination of the fluorotic enamel results reveals that, although the fluorotic enamel is more acid-resistant, the prolonged application mode improves the demineralization, which appears as an increase in micro-irregularities and prism porosities in the microscopy analysis. Further, the mode also improves the interaction of acidic monomers with prismatic and interprismatic areas [6].

However, there was no significant difference between the all-etching bond system and the self-etching bond system for moderate and severe fluorosis, possibly because the degree of demineralization of the enamel in the moderate and severe fluorosis was low, and the enamel was more fragile and easier to break [29]. Various studies have reported that the prolonged application time of universal adhesives in SE mode to a fluorotic enamel enhances the enamel-etching pattern and promotes the similarity of the results in terms of adhesive enamel bond strength [6].

\section{Conclusions}

This study found that the bonding strength of Prime \& Bond NT for an enamel with mild fluorosis was better than that of SE-Bond, and cohesive failure was observed as the most common mode of failure. Additionally, there was no difference in the bonding strength of the SE-Bond for different DF degrees. Therefore, we recommend the use of the all-etching adhesive system in the clinical treatment of teeth with mild fluorosis.

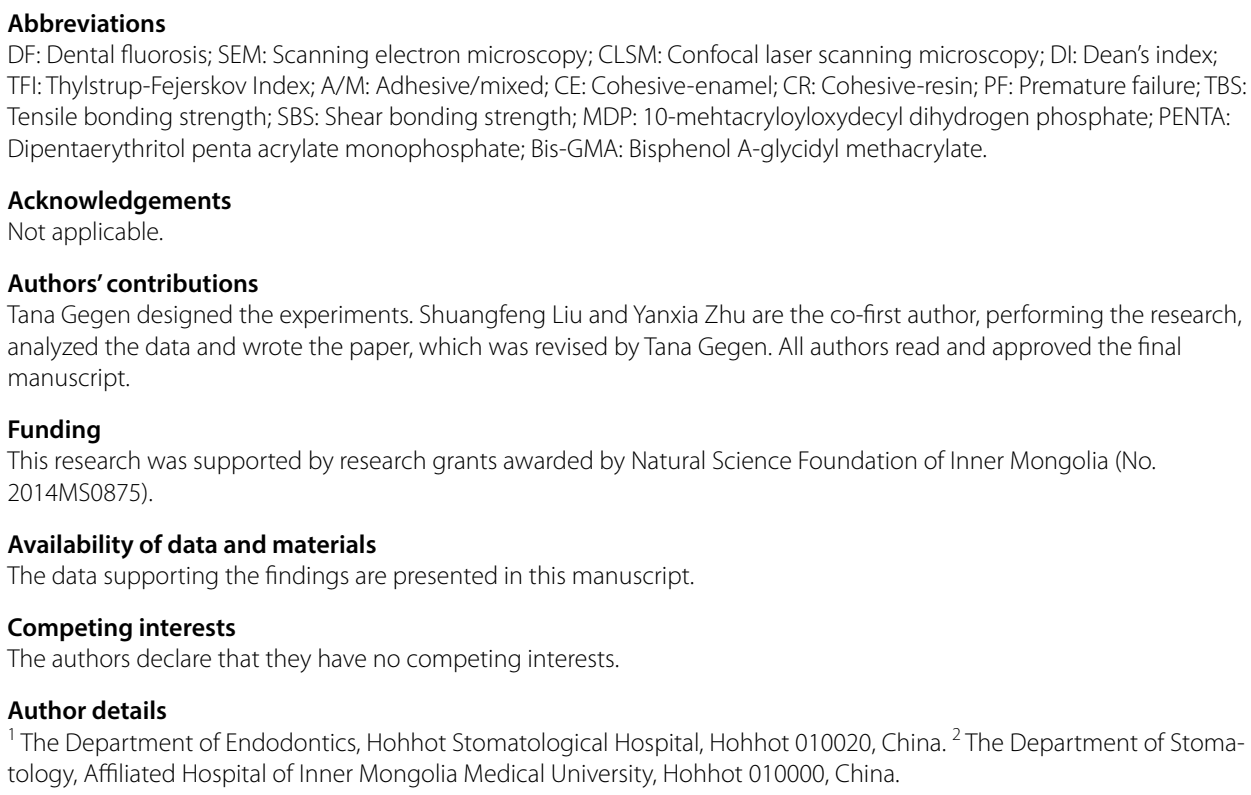

Received: 4 March 2020 Accepted: 12 August 2020

Published online: 19 August 2020 


\section{References}

1. Mascarenhas AK. Risk factors for dental fluorosis: a review of the recent literature. J Pediatr Dent. 2000;22:269-77.

2. Zhao LJ, Pei JR, Zhang W, et al. Surveillance on drinking-water-born endemic fluorosis in China, 2013. Zhonghua liu xing bing xue za zhi. 2016;37:816-20.

3. Yuan L, Fei W, Jia F, et al. Health risk in children to fluoride exposure in a typical endemic fluorosis area on Loess Plateau, north China, in the last decade. J Chemosphere. 2020;243:125451.

4. Thylstrup A, Fejerskov O. Clinical appearance of dental fluorosis in permanent teeth in relation to histologic changes. Comm Dent Oral Epidemiol. 1978;6:315-28.

5. Akpata ES. Occurrence and management of dental fluorosis. Int Dent J. 2001;51:325-33.

6. Siqueira FSF, Armas-Vega A, Izquierdo-Bucheli A, et al. Does the conditioning mode and duration of universal adhesives affect the bonding effectiveness to fluorotic enamel? J Adhes Dent. 2019;21:525-36.

7. El Mourad AM. Aesthetic rehabilitation of a severe dental fluorosis case with ceramic veneers: a step-by-step guide. Case Rep Dent. 2018;2018:4063165.

8. Schoppmeier CM, Derman SHM, Noack MJ, et al. Power bleaching enhances resin infiltration masking effect of dental fluorosis a randomized clinical trial. J Dent. 2018;79:77-84.

9. Sundfeld D, Pavani CC, Pavesi Pini NI, et al. Esthetic recovery of teeth presenting fluorotic enamel stains using enamel microabrasion and home-monitored dental bleaching. J Conserv Dent. 2019;22:401-5.

10. Christensen GJ. Clinical factors affecting adhesion. Oper Dent. 1992;Suppl 5:24-31.

11. Ateyah N, Akpata E. Factors affecting shear bond strength of composite resin to fluorosed human enamel. Oper Dent. 2000;25:216-22.

12. Torres-Gallegos I, Martinez-Castañon GA, Loyola-Rodriguez JP, et al. Effectiveness of bonding resin-based composite to healthy and fluorotic enamel using total-etch and two self-etch adhesive systems. Dent Mater J. 2012:31:1021-7.

13. Tiwari P, Kaur S, Sodhi A. Dental fluorosis and its association with the use of fluoridated toothpaste among middle school students of Delhi. Indian J Med Sci. 2010;64:1-6.

14. Dean HT. Fluorine and dental caries; epidemiological aspects. J Dent Res. 1947;26:339.

15. Mabelya L, van'tHof MA, König KG, et al. Comparison of two indices of dental fluorosis in low, moderate and high fluorosis Tanzanian populations. Commun Dent Oral Epidemiol. 1994;22:415-20.

16. Okuda M, Pereira PN, Nikaido T, et al. Evaluation of in vitro secondary caries using confocal laser scanning microscope and X-ray analytical microscope. Am J Dent. 2003;16:191-6.

17. Ramesh M, Narasimhan M, Krishnan R, et al. The effect of fluorosis on human teeth under light microscopy: a crosssectional study. J Oral Maxillofac Pathol. 2017;21:345-50.

18. Gu M, Lv L, He X, et al. Effect of phosphoric acid concentration used for etching on the microtensile bond strength to fluorotic teeth. Med. 2018;97:e12093.

19. Yanagisawa T, Takuma S, Fejerskov O. Ultrastructure and composition of enamel in human dental fluorosis. Adv Dent Res. 1989;3:203-10.

20. Waidyasekera PG, Nikaido T, Weerasinghe DD, et al. Bonding of acid-etch and self-etch adhesives to human fluorosed dentine. J Dent. 2007;35:915-22.

21. Li HS. A Study on bonding strength of orthodontic brackets to fluorosed teeth. Qingdao: Qingdao University; 2009.

22. Torres-Méndez F, Martinez-Castañon GA, Torres-Gallegos I, et al. Effects of silver nanoparticles on the bonding of three adhesive systems to fluorotic enamel. Dent Mater J. 2017;36:266-74.

23. Weerasinghe DS, Nikaido T, Wettasinghe KA, et al. Micro-shear bond strength and morphological analysis of a selfetching primer adhesive system to fluorosed enamel. J Dent. 2005:33:419-26.

24. Hui HW, Qi SQ. Clinical observation on the effect of two kinds of orthodontic adhesives for orthodontic dental fluorosis. J Modern Prev Med. 2011;38:600.

25. Fejerskov O, Manji F, Baelum V. The nature and mechanisms of dental fluorosis in man. J Dent Res. 1990;69:692-700.

26. Priyadharsini N, Malathi N, Tamizhchelvan H, et al. Dental fluorosis: a histological study using Light and Confocal microscopy. Indian J Dent Res. 2015;26:248-51.

27. Holmen L, Thylstrup A, Featherstone JD, et al. A scanning electron microscopic study of surface changes during development of artificial caries. Caries Res. 1985;19:11-21.

28. Mcleod ME, Price RB, Felix CM. Effect of configuration factor on shear bond strengths of self-etch adhesive systems to ground enamel and dentin. Oper Dent. 2010;35:84-93.

29. Waidyasekera PG, Nikaido T, Weerasinghe DD, et al. Caries susceptibility of human fluorosed enamel and dentine. J Dent. 2007:35:343-9.

30. Li N, Nikaido T, Alireza S, et al. Phosphoric acid-etching promotes bond strength and formation of acid-base resistant zone on enamel. Oper Dent. 2013:38:82-90.

31. Opinya GN, Pameijer CH. Tensile bond strength of fluorosed Kenyan teeth using the acid etch technique. Int Dent J. 1986;36:225-9.

\section{Publisher's Note}

Springer Nature remains neutral with regard to jurisdictional claims in published maps and institutional affiliations. 